\title{
Reduction of Closed Frontal Sinus Fractures through Suprabrow Approach
}

\author{
Hyung Min Hahn, \\ Yoo Jung Lee, \\ Myong Chul Park, \\ Il Jae Lee, \\ Sue Min Kim, \\ Dong Ha Park \\ Department of Plastic and Reconstructive \\ Surgery, Ajou University School of Medicine, \\ Suwon, Korea
}

\begin{abstract}
Background: The traditional approach for reduction of frontal sinus fractures is coronal incision. Inherent complications of the coronal approach include long scar, hair loss, and long operation time. We describe a simple approach for the reduction of frontal sinus anterior wall fractures using a suprabrow incision that is commonly used for brow lift.

Methods: From March 2007 to October 2016, the authors identified patients with anterior wall frontal sinus fractures treated by open reduction through a suprabrow incision. Only cases with photographic/radiographic documentation and a minimum follow-up of 6 months were included. The incision line was designed to be at the upper margin of the eyebrow. Medical records and radiographic data were retrospectively reviewed. Surgical outcomes, cosmetic results, and complication were assessed. The patient scale of the patient and observer scar assessment scale was used to assess patient satisfaction for incisional scar at the 6-month follow-up.

Results: Thirty-one patients underwent fracture reduction through a suprabrow approach during the study period, with a mean follow-up of 41 months. No patients showed any recurrent displacement, eyebrow asymmetry, or infection during follow-up. Thirteen patients reported their forehead paresthesia postoperatively, and 12 of them had preoperative symptom. One patient complained of incisional scar and underwent scar revision. All patients were satisfied with their eyebrow and forehead contour.

Conclusion: The suprabrow approach allowed for an accurate reduction of the fractures in the anterior wall frontal sinus by providing direct visualization of the fracture. This transcutaneous approach can effectively restore forehead contour with acceptable postoperative complications and patient satisfaction.
\end{abstract}

Keywords: Frontal sinus / Eyebrows / Forehead / Skull fractures / Minimally invasive surgical procedures

\section{INTRODUCTION}

As the frequency of high-speed motor vehicle accidents and violent instrument-aided assaults rises, the incidence of facial trauma is increasing [1]. Frontal sinus fractures are less common than other facial bone fractures, but they comprise $5 \%$ to $12 \%$ of all maxillofacial fractures [2,3]. Frontal sinus fractures pose a signifi-

\section{Correspondence: Dong Ha Park}

Department of Plastic and Reconstructive Surgery, Ajou University School of Medicine, 164 World cup-ro, Yeongtong-gu, Suwon 16499, Korea

E-mail: growhand@hanmail.net

Received Sep 8, 2017 / Revised Oct 18, 2017 / Accepted Oct 18, 2017 cant challenge. The proper treatment paradigm has been a center of debate for many years. Immediate concerns include protection of intracranial structures, identification of associated injuries, and control of cerebrospinal fluid leakage. In some of the frontal sinus fracture that involves only the anterior table, the aesthetic forehead contour is an important consideration in repair. Newer, modified algorithms incorporate these technologic advancements for improved functional and cosmetic results. Several efforts were reported to reduce the potential morbidities of coronal approach using endoscopic technique [4-7] or transcutaneous approach [8-12]. These approaches allow adequate visualization of 
the fracture site with acceptable postoperative course, which may be avoided by use of minimally-invasive techniques.

We describe a simple approach with the aim of minimizing the complications of a coronal incision and decreasing operation time, within the boundary of acceptable cosmetic outcomes.

\section{METHODS}

From March 2007 to October 2016, the authors identified patients with closed fractures of anterior wall frontal sinus treated by open reduction through a suprabrow incision. Open fractures, closed fractures treated by noninvasive methods, and fractures with complete naso-frontal duct damage were excluded. In complete naso-frontal duct obstruction, we chose the bicoronal approach for the sinus obliteration. For incomplete or absent naso-frontal duct obstruction, we chose the bicoronal approach in large fracture area over than $40 \mathrm{~mm} \times 30 \mathrm{~mm}$ (Fig. 1). Only cases with photographic/radiographic documentation and a minimum follow- up of 6 months were included. Preoperative and postoperative computed tomography (CT) scans were obtained with three-dimensional reconstruction.

Medical records and radiographic data were retrospectively reviewed. Clinical variables that were recorded included patient age, sex, mechanism of injury, interval time between injury and surgery, operation time, incision length, methods of fixation, associated fractures, preoperative sensory deficit, and width of fragments. Recorded clinical outcomes were postoperative complication, additional procedure related to the fracture, forehead hypoesthesia, postoperative radiologic assessment of reduction, eyebrow symmetry and movement, and patient satisfaction for incisional scar. The postoperative complications that were assessed included wound dehiscence, wound infection, hematoma, sinusitis, mucocele, and plate migration. Fracture reduction was evaluated through postoperative axial images of CT scans by comparing the reduced and contralateral sides. Patients reported their subjective assessment of incisional scar at the 6-month follow-up

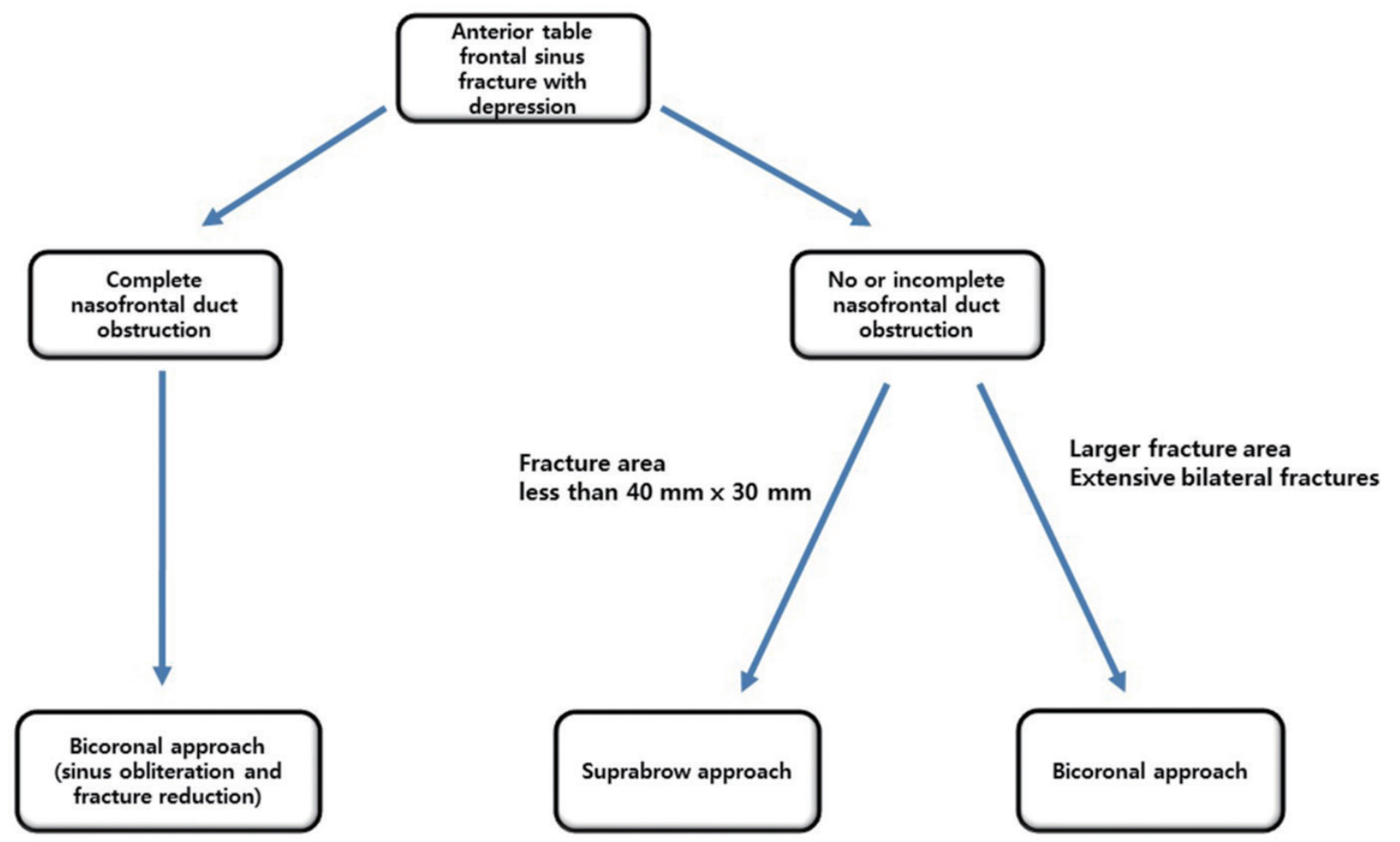

Fig. 1. Algorithm of reduction of frontal sinus fractures. 
visit using the patient scale from the patient and observer scar assessment scale (POSAS) [13]. The questionnaire included pain, itching, color, stiffness, thickness, and irregularity of the scar. Each of the six parameters were rated from 1 to 10 , with 10 as the worst outcome. The total score consists of adding the scores of each of the six items (range, 6 to 60 ). In addition to eyebrow scar, eyebrow symmetry and movement were assessed with photographic documentation at the follow-up visit. Bilateral eyebrow position was measured on the preoperative and postoperative clinical photographs. Patients with preoperative eyebrow asymmetry were excluded from the analysis. Eyebrow asymmetry was defined bilaterally as more than $2 \mathrm{~mm}$ difference of vertical distance from the interpupillary line to the superior margin of the eyebrow on photographic evaluation. Photographs were taken with a natural gaze and at bilateral brow elevation with the forehead wrinkled.

\section{Surgery}

The surgery was performed with the patient in a supine position under general anesthesia. A line was drawn along the upper margin of the eyebrow between the both ends of the eyebrow to obtain an inconspicuous scar (Fig. 2). Eyebrow shape needed to be taken into account, as there was a slight difference between male and female eyebrows. The brows are flatter and are lower along the orbital rim in males compared with females [14]. After infiltration of 2\% lidocaine with epinephrine, an incision was made using a \#15 blade, maintaining the blade of the scalpel angled but parallel to the axis of the follicles to preserve them. Dissection was deepened to incise orbital orbicularis and frontalis muscles horizontally. Care was taken not to injure the supratrochlear and supraorbital nerves, which run below the subcutaneous muscular aponeurotic system in this surgical field [15-17] (Fig. 3). Meticulous muscle dissection was needed in the supraorbital region, as orbicularis and frontalis muscles are blended, especially in medial attachment. A periosteal incision with blade was made near the edge of the fracture site and limited subperiosteal dissection was performed towards the edge to preserve the periosteal attachment on the anterior table of the frontal sinus. The fractured segment of the anterior table of the frontal sinus was then reduced gently by pushing with the elevator and bone hook. The sinus mucosa and periosteum attached to the fractured fragments were preserved to maintain the reduced position and to avoid flap-to-graft conversion of the fracture segments. After the reduction was confirmed, fractured bones were fixed using plates and screws (Fig. 3).

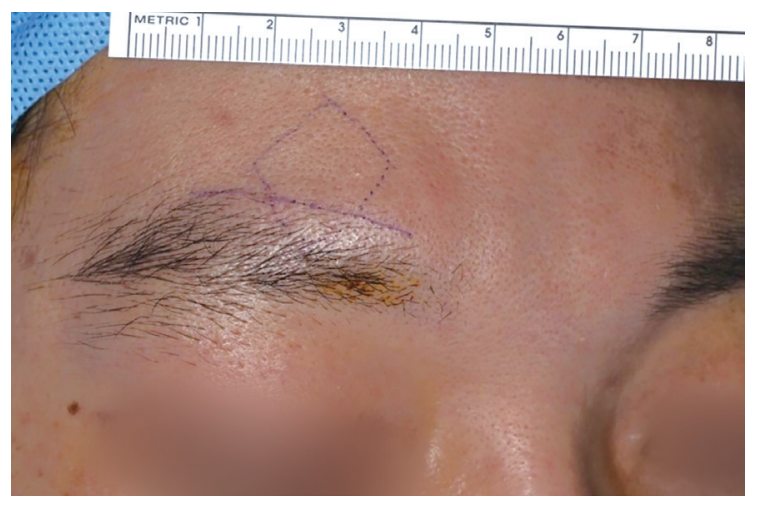

Fig. 2. Design of skin incision. A line is drawn along the upper margin of the eyebrow between the both ends of the eyebrow.
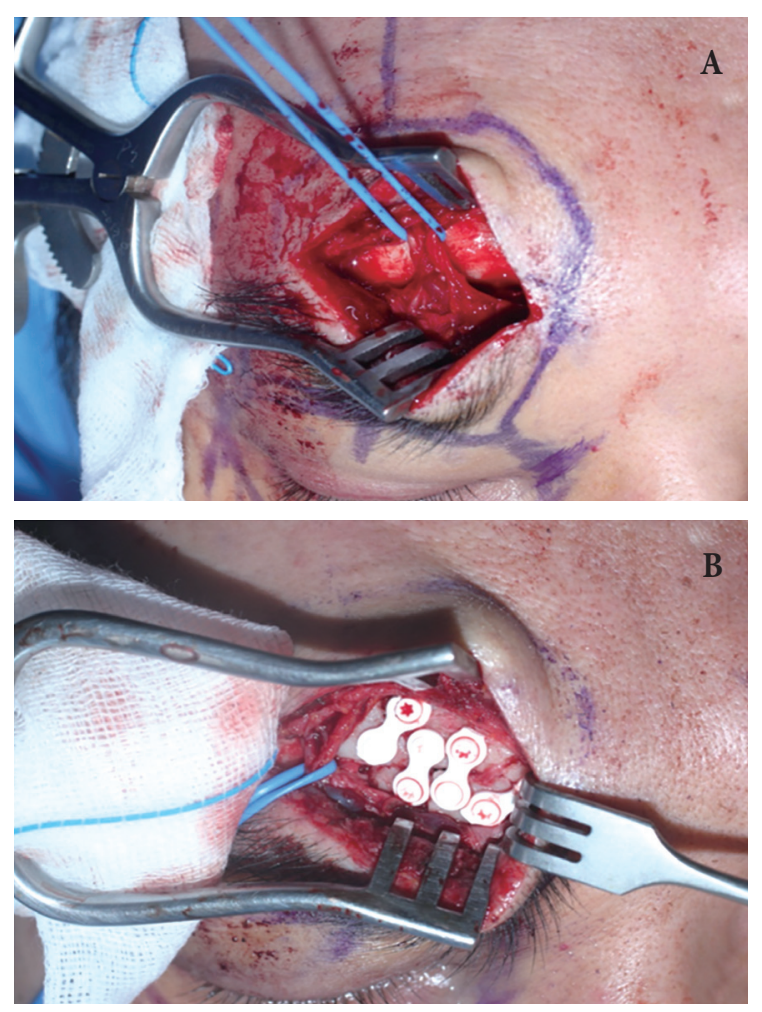

Fig. 3. Intraoperative findings. (A) During operation, supratrochlear and supraorbital nerves were preserved. (B) After reduction of fractured segments, fractured bones were fixed using plates and screws. 
The periosteum was closed to cover an exposed plate or mesh and to avoid wound infection. The surgical site was irrigated and skin-incision wounds were carefully closed layer by layer with absorbable and non-absorbable sutures. Care was taken at this time to minimize hair-follicle injuries. No pressure dressing or drains were placed. A CT scan was obtained immediately following surgery to evaluate the operative outcome. To drain the frontal sinus, the head was slightly elevated for several days after the operation.

\section{Statistics}

Descriptive statistics were calculated, including frequencies for categorical variables and means, standard deviations, and ranges for continuous variables. The values for the numbers of cases, area, length, and scores are expressed as original amount and percentage to one decimal place. Years, months, days and minutes were rounded up to the nearest integer numbers.

\section{RESULTS}

Patient ages ranged from 13 to 78 years, with a mean age of 32 years. Patient demographics and injury characteristics are summarized in Table 1. Most cases resulted from sports injury, road traffic accident, physical assault, or some other form of blunt trauma. The locations of fracture were right side in 15 cases, left side in 14 cases, and both side in two cases. The mean fractured area was $676.8 \pm 340.8 \mathrm{~mm}^{2}$. Other facial fractures were combined in 14 cases. One case was combined with zygomaticomaxillary complex fracture and nasal bone fracture. One case was combined with orbital floor blowout fracture and nasal bone fracture. Other cases were nasal bone fracture $(n=9)$, orbital floor blowout fracture $(n=2)$, and naso-ethmoid-orbital fracture $(n=1)$. Hypoesthesia on forehead before the operation was noted in 12 patients.

Surgery was performed within 2 weeks of injury in all patients. Indication of the operation was forehead contour deformity in all cases. The results of surgery are summarized in Table 2 . The mean operation time was $46 \pm 21$ minutes. The mean length of surgical incision was $2.5 \pm 0.6 \mathrm{~cm}$. The fixation was performed in 24 cases. The fractured segments were fixed with metal plates in 16 cases
Table 1. Patient demographics and injury characteristics

\begin{tabular}{lc} 
Variable & Number $(\%)$ \\
Age $(y r)$ & $31.9 \pm 14.8$ \\
Sex & \\
Male & $29(93.5)$ \\
Female & $2(6.5)$ \\
Mechanism of injury & \\
Sports injury & $10(32.3)$ \\
Road traffic accident & $7(22.6)$ \\
Physical assault & $6(19.4)$ \\
Falling & $2(6.5)$ \\
Others & $6(19.4)$ \\
Sensory nerve injury & $12(38.7)$ \\
Fracture characteristics & \\
Location & \\
Right & $15(48.4)$ \\
Left & $14(45.2)$ \\
Both & $2(6.5)$ \\
Fracture area (mm²) & $676.8 \pm 340.8$ \\
Concurrent facial fractures & \\
Nasal bone & $11(35.5)$ \\
Orbital floor blowout & $3(9.7)$ \\
Zygomaticomaxillary complex & $1(3.2)$ \\
Naso-ethmoid-orbit & $1(3.2)$ \\
None & $17(54.8)$ \\
\hline
\end{tabular}

Values are presented as mean \pm standard deviation or number (\%).

Table 2. Results of surgery

\begin{tabular}{lc} 
Variable & Number (\%) \\
\hline Interval from trauma to surgery (day) & $7.1 \pm 3.2$ \\
\hline Operation time (min) & $46.3 \pm 21.0$ \\
\hline Incisional length (cm) & $2.5 \pm 0.6$ \\
Fixation material & \\
Metal plate & $16(51.6)$ \\
Absorbable plate & $2(6.5)$ \\
Absorbable mesh plate & $6(19.4)$ \\
None & $7(22.6)$ \\
Follow-up period (mo) & $41.1 \pm 19.3$ \\
Complications & $1(3.2)$ \\
Forehead hypoesthesia, postoperatively & \\
$\quad$ developed & $1(3.2)$ \\
Hypertrophic scar & $9.6 \pm 1.6$ \\
\hline Total score of patient scale of POSAS (6-60)
\end{tabular}

Values are presented as mean \pm standard deviation or number (\%).

POSAS, patient and observer scar assessment scale.

and with absorbable mesh plate in 6 cases. Two cases were fixed with absorbable plates.

Preoperative CT scans showed no evidence of naso-frontal duct obstruction in any of the cases. Access to fracture site of the frontal sinus through the incision was possible in all cases. In two cases, postoperative assessment of fracture reduction in CT axial images revealed a $2 \mathrm{~mm}$ depression on the edge of fracture segment compared to anterior wall of contralateral frontal sinus. In other cases, postoperative CT scans showed adequate reduction 
of displaced bone fragments (Fig. 4).

Follow-up duration ranged from 6 to 60 months, with an average of 41 months. No complications related to this procedure, such as uncontrollable sinus bleeding, infection, any recurrent displacement, and posterior table and brain injuries, were observed. Thirteen patients reported their forehead paresthesia postoperatively; 12 had the symptom preoperatively and the symptom developed postoperatively in 1 patient. Other patients had improvement of the hypoesthesia, however, two patients who had preoperative numbness of their forehead suffered from continuous hypoesthesia in long-term follow-up.
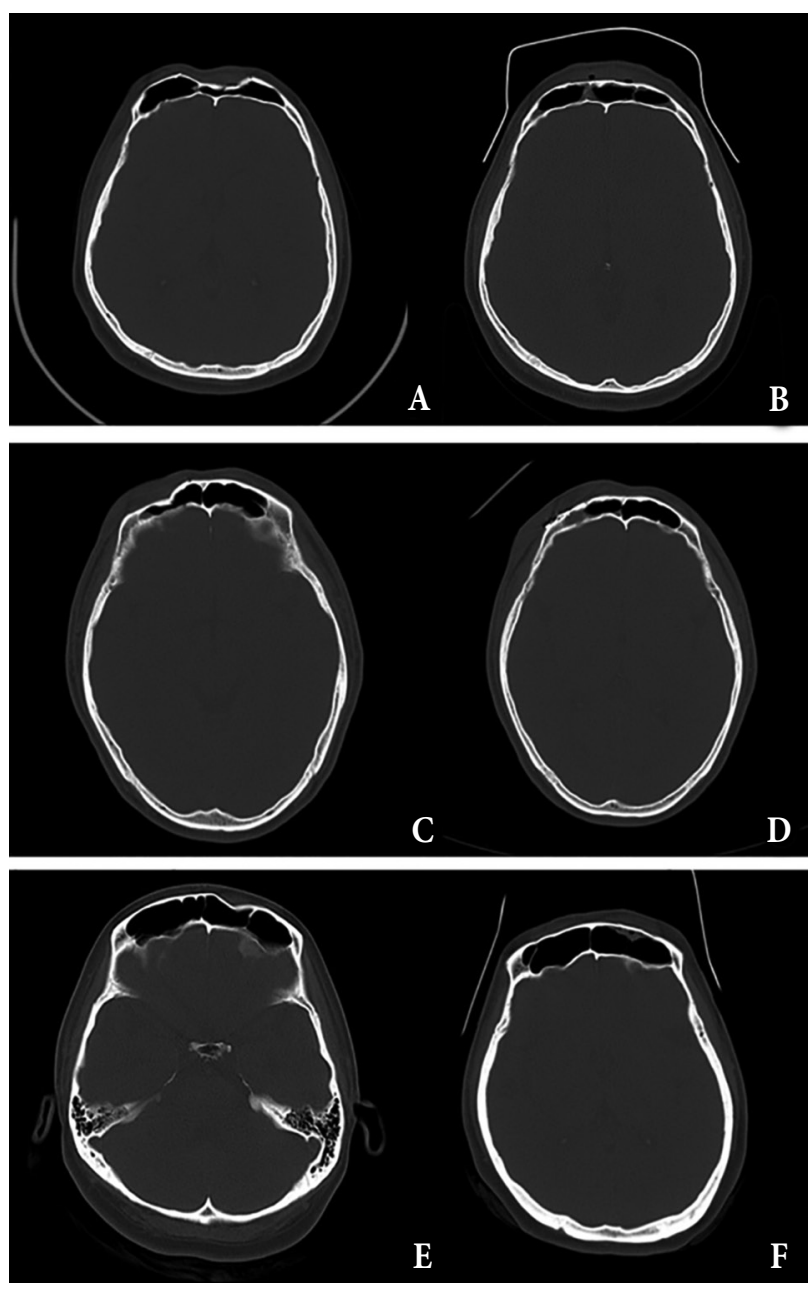

Fig. 4. Preoperative and postoperative CT scans. (A, C, E) Preoperative CT scans showed depressed anterior table of frontal sinus. (B, D, F) Postoperative CT scans showed restored contour of anterior table of frontal sinus by open reduction through suprabrow incision. CT, computed tomography.
Forehead contours were effectively restored in all cases. No patient required further operation related to contour correction. Two patients who showed bony depression on postoperative CT scan had symmetric forehead contours at the follow-up visit. The cosmetic results of the incisional scar were acceptable. At the 6-month follow-up, all patients were asked to answer the questionnaire regarding the incisional scar using the POSAS tool. The mean total scores were 9.6 (range, 7-13). One patient complained of the incisional scar, and underwent scar revision after 6 months of frontal sinus reconstruction.

In the evaluation of the eyebrow symmetry and movement, five patients had preoperative eyebrow asymmetry and so were excluded from the photographic analysis. All 26 patients achieved symmetric eyebrow level and movement at postoperative followup (Fig. 5). No patient complained of discomfort in eyebrow elevation and forehead wrinkle formation.

\section{DISCUSSION}

The surgical approach to closed anterior table fractures is continuously evolving. The primary goal is to achieve adequate reduction of the fracture with restoration of contour. Management should focus on protection of the intracranial content against early and delayed complications, restoration of the upper third facial contour, and cessation of cerebrospinal fluid. The conventional surgical approach to the anterior table of the frontal sinus is an open approach through a coronal incision. For a non-displaced anterior table, frontal sinus fractures pose a minimal risk of mucocele formation or contour deformity. These non-displaced fractures can be managed expectantly.

The bicoronal approach is the traditional approach for frontal sinus surgery, especially for obliteration or cranialization. Drawbacks of the approach include long visible scar, alopecia along the incision line, injury to the frontal branch of the facial nerve, long operation time, prolonged hospital stay due to wide area of dissection and risk for postoperative bleeding [15]. Male patients concerned about alopecia along the long scar prefer the suprabrow incision to the bicoronal incision. In our cases of suprabrow incision, the incisional scar was aesthetically acceptable for most patients 

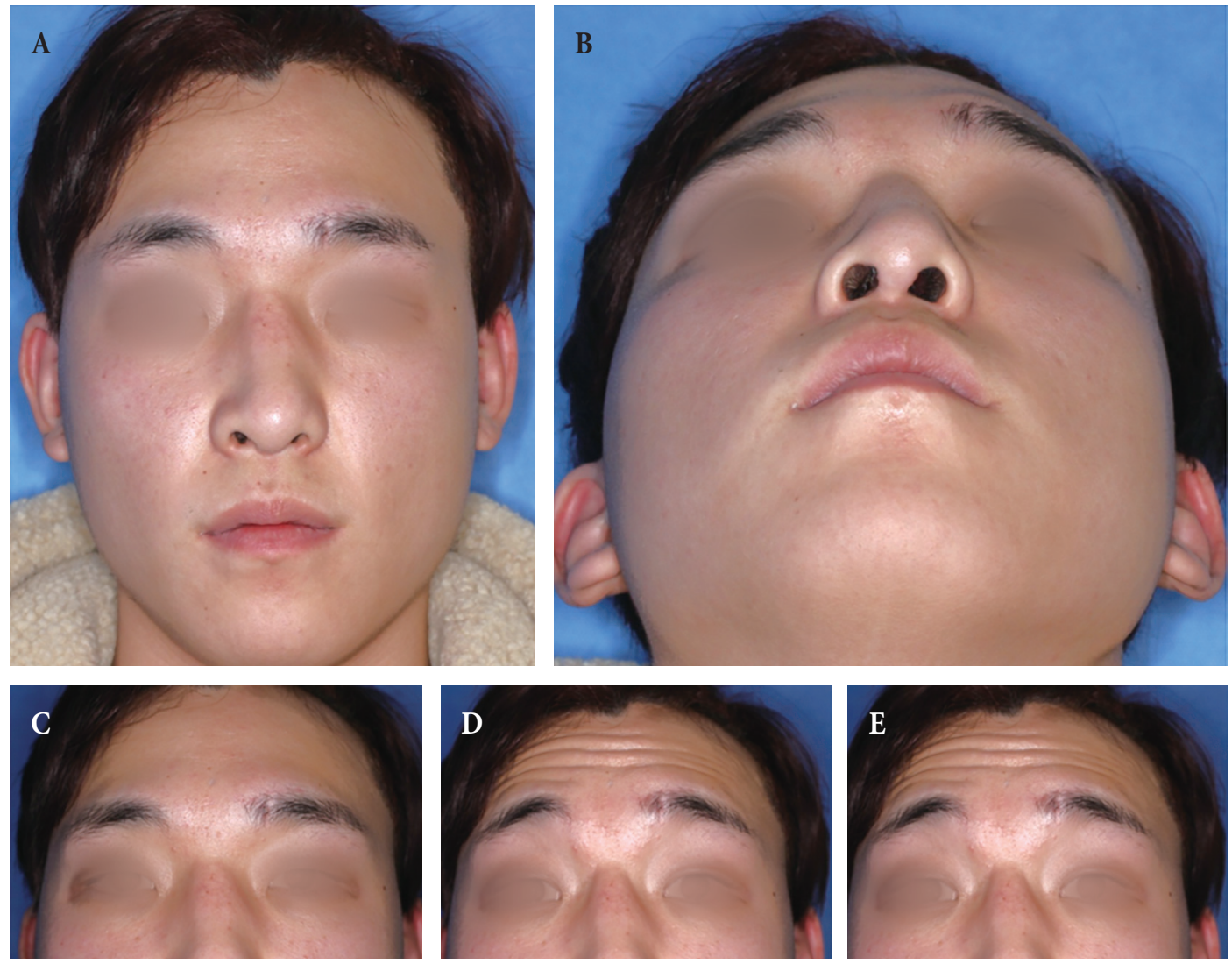

Fig. 5. (A-E) Postoperative aesthetic results. A 23-year-old male with left anterior table fracture of the frontal sinus. The reduction of frontal sinus was performed using the suprabrow incision. At six weeks postoperatively, the forehead contour was restored and the scar was aesthetically acceptable. There was no asymmetry in eyebrow height and movement.

(mean POSAS total score 9.6). Also, there was no impairment in eyebrow elevation and asymmetry.

Using endoscopic procedure in repair of frontal sinus fracture was first described by Graham and Spring [4] in 1996. After that, several studies were documented using endoscopic technique with minimal frontal scars and avoidance of morbidities associated with coronal flap [5-7]. However, this endoscopic technique has disadvantages of limited field of view and steep learning curve.

Several transcutaneous approaches were reported and had distinctive advantages and disadvantages. Using a small peri-brow incision [9] or a slit incision [10], aesthetic results were better than other surgical approaches but rigid internal fixation was impossible. Through a frontalis rhytid forehead incision [11], satisfactory forehead contour and fracture reduction were achieved with aes- thetically favorable scar appearance. However, this incision is not applicable to young patients whose frontalis rhyid is not visible. Subbrow approach has advantages similar to our suprabrow approach in regard to direct visualization of the fracture site and inconspicuous scar [12]. Our suprabrow approach has an extensive visualization of the fracture and enables an accurate reduction and rigid fixation because the incision located much closer to the frontal sinus than subbrow approach. Contrary to other transcutaneous approaches, our suprabrow technique has advantages of direct visualization of fractured site, possibility of rigid fixation, and aesthetically acceptable scars.

Although this transcutaneous approach does not require technical complexity for facial surgeons, anatomical variations need to be taken into account, since neural and vascular structures underneath the skin incision could interfere with fracture reduction. 
In the brow and glabella area, supratrochlear and supraorbital vessels and nerves exit the orbit. The supratrochlear artery is at the vertical line from medial canthus with slightly medial deviation. At the level of the brow, the artery passes deep to the orbicularis and frontalis [14]. Approximately 15 to $25 \mathrm{~mm}$ above the orbital rim, the artery traverses the frontalis and orbicularis to enter the subcutaneous plane [14]. Although there is some variation in the exit point of supraorbital nerve [16], this point of emergence is approximately 25 to $30 \mathrm{~mm}$ from the midline. The nerve then divides into a superficial (medial) and a deep (lateral) branch. The supraorbital artery emerge from the orbit through the supraorbital notch approximately $32 \mathrm{~mm}$ lateral to midline, generally corresponding to a vertical line from the medial limbus [17].

The mean size of frontal sinus measured in a prior study was $68 \mathrm{~mm}$ for latero-lateral and $39 \mathrm{~mm}$ for supero-inferior distances [18]. Although both frontal sinuses are not symmetric in most cases, patients with unilateral fracture can be managed with a transcutaneous incision in a case with fracture width less than 40 $\mathrm{mm}$. Presently in the two bilateral fractures, the fracture segments were not so extensive that would cross the glabella midline. Skin incision extending over medial border of eyebrow should be avoided for aesthetic reasons. Instead, the bicoronal approach is preferable in extensive bilateral frontal sinus fractures.
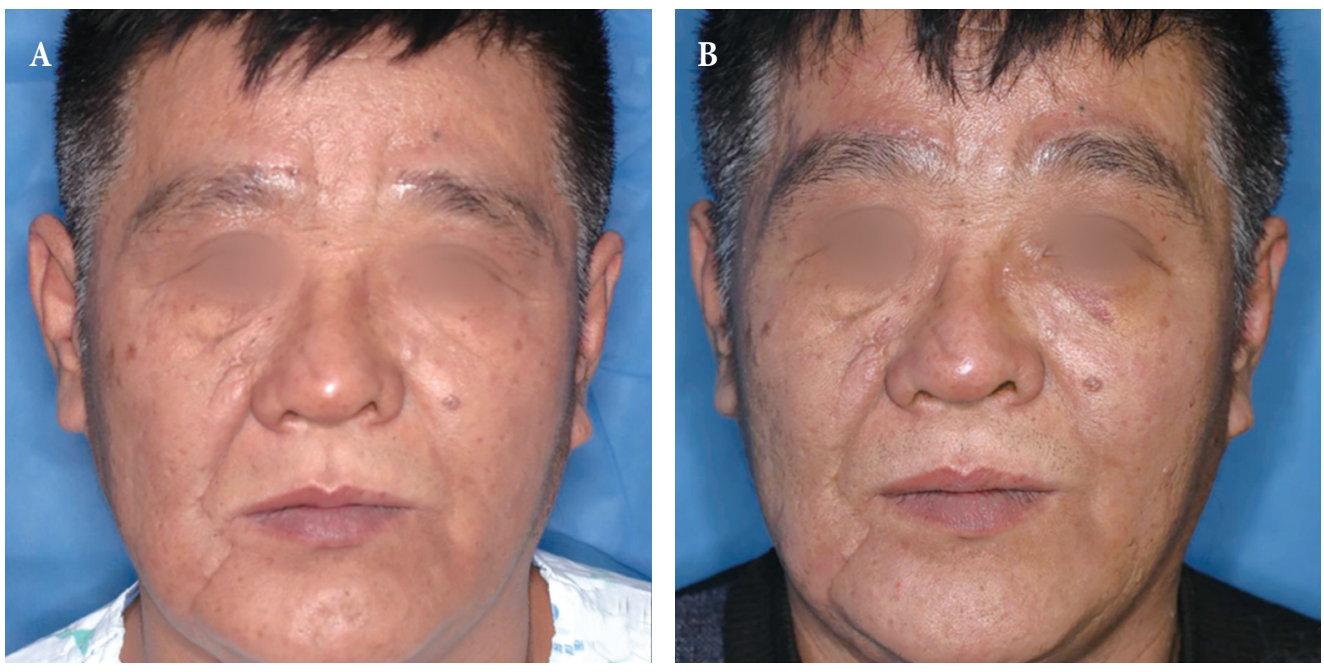

Fig. 6. Patient with brow ptosis. (A) Patient with brow ptosis on both sides. (B) After direct brow lift with suprabrow incision, the location of the eyebrow was improved (2 weeks postoperatively). 
can effectively restore the forehead contour, while allowing acceptable postoperative complications and patient satisfaction. Traumatized patients, especially older patients who want to receive a brow lifting surgery combined with operation for frontal fracture under one time of general anesthesia, can benefit from this approach.

\section{CONFLICT OF INTEREST}

No potential conflict of interest relevant to this article was reported.

\section{PATIENT CONSENT}

The patient provided written informed consent for the publication and the use of their images.

\section{REFERENCES}

1. Lim JS, Oh YH, Cho SP, Han KT, Lim P. A clinical review of frontal sinus fracture. J Korean Soc Plast Reconstr Surg 1991;18:274-80.

2. Rohrich RJ, Hollier LH. Management of frontal sinus fractures: changing concepts. Clin Plast Surg 1992;19:219-32.

3. Tiwari P, Higuera S, Thornton J, Hollier LH. The management of frontal sinus fractures. J Oral Maxillofac Surg 2005;63:1354-60.

4. Graham HD 3rd, Spring P. Endoscopic repair of frontal sinus fracture: case report. J Craniomaxillofac Trauma 1996;2:52-5.

5. Lappert PW, Lee JW. Treatment of an isolated outer table frontal sinus fracture using endoscopic reduction and fixation. Plast Reconstr Surg 1998;102:1642-5
6. Strong EB, Kellman RM. Endoscopic repair of anterior table: frontal sinus fractures. Facial Plast Surg Clin North Am 2006;14:25-9.

7. Strong EB. Endoscopic repair of anterior table frontal sinus fractures. Facial Plast Surg 2009;25:43-8.

8. Hwang K, Song YB. Closed reduction of fractured anterior wall of the frontal bone. J Craniofac Surg 2005;16:120-2.

9. Kim KS, Kim ES, Hwang JH, LeeSY. Transcutaneous transfrontal approach through a small peri-eyebrow incision for the reduction of closed anterior table frontal sinus fractures. J Plast Reconstr Aesthet Surg 2010;63:763-8.

10. Yoo A, Eun SC, Baek RM. Transcutaneous reduction of frontal sinus fracture using bony tapper device. J Craniofac Surg 2012;23:1835-7.

11. Noury M, Dunn RM, Lalikos JF, Fudem GM, Rothkopf DM. Frontal sinus repair through a frontalis rhytid approach. Ann Plast Surg 2011;66:457-9.

12. Kim J, Choi H. A review of subbrow approach in the management of non-complicated anterior table frontal sinus fracture. Arch Craniofac Surg 2016;17:186-9.

13. Draaijers LJ, Tempelman FR, Botman YA, Tuinebreijer WE, Middelkoop E, Kreis RW, et al. The patient and observer scar assessment scale: a reliable and feasible tool for scar evaluation. Plast Reconstr Surg 2004;113:1960-5.

14. Gunter JP, Antrobus SD. Aesthetic analysis of the eyebrows. Plast Reconstr Surg 1997;99:1808-16.

15. Godhi SS, Kukreja P, Singh V, Goyal S. Versatility of the coronal approach in maxillofacial surgery. J Oral Health Community Dent 2010;4:16-21

16. Janis JE, Ghavami A, Lemmon JA, Leedy JE, Guyuron B. The anatomy of the corrugator supercilii muscle: part II. Supraorbital nerve branching patterns. Plast Reconstr Surg 2008;121:233-40.

17. Kleintjes WG. Forehead anatomy: arterial variations and venous link of the midline forehead flap. J Plast Reconstr Aesthet Surg 2007;60:593-606

18. Rubira-Bullen IR, Rubira CM, Sarmento VA, Azevedo RA. Frontal sinus size on facial plain radiographs. J Morphol Sci 2010;27:77-81. 